\title{
Correction to: Anoikis resistant gastric cancer cells promote angiogenesis and peritoneal metastasis through C/EBP $\beta$-mediated PDGFB autocrine and paracrine signaling
}

Shangce Du (D, Zhi Yang, Xiaofeng Lu, Suhail Yousuf, Min Zhao, Wenxi Li, Ji Miao, Xingzhou Wang, Heng Yu, Xinya Zhu, Hong Chen, Linseng Shi, En Xu (D), Xuefeng Xia (D) and Wenxian Guan (iD)

(c) The Author(s), under exclusive licence to Springer Nature Limited 2021

Oncogene (2022) 41:1384-1385; https://doi.org/10.1038/s41388-021-02096-7

Correction to: Oncogene (2021) 40:5764-5779; https://doi.org/ 10.1038/s41388-021-01988-y, published online 2 August 2021

Following the publication of this article the authors noted the images for group-AGS-AR GC CM in figure $3 F$ and group-AGS ARsiRNA PDGFB in Figure 6F were inadvertently misplaced. The corrected images are provided below: 

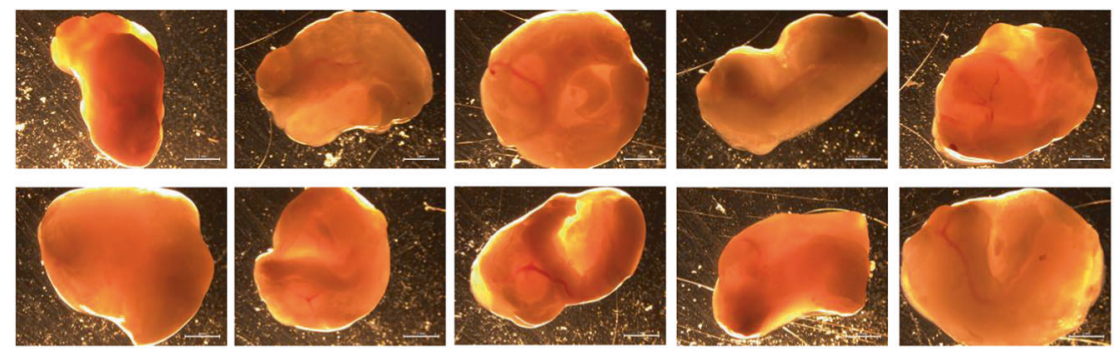

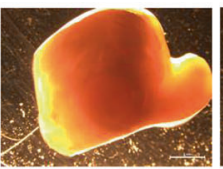

Ctrl CM

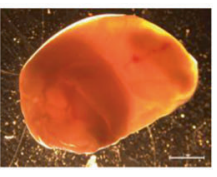

Normal GC CM

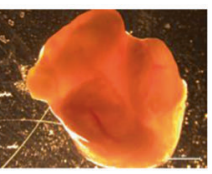

AR GC CM

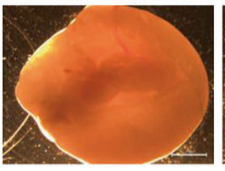

$A R$ GC CM + PDGFBAb

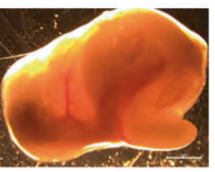

AR GC+CXCL11 Ab

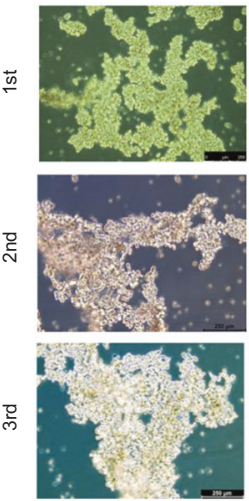

siRNA-Vector
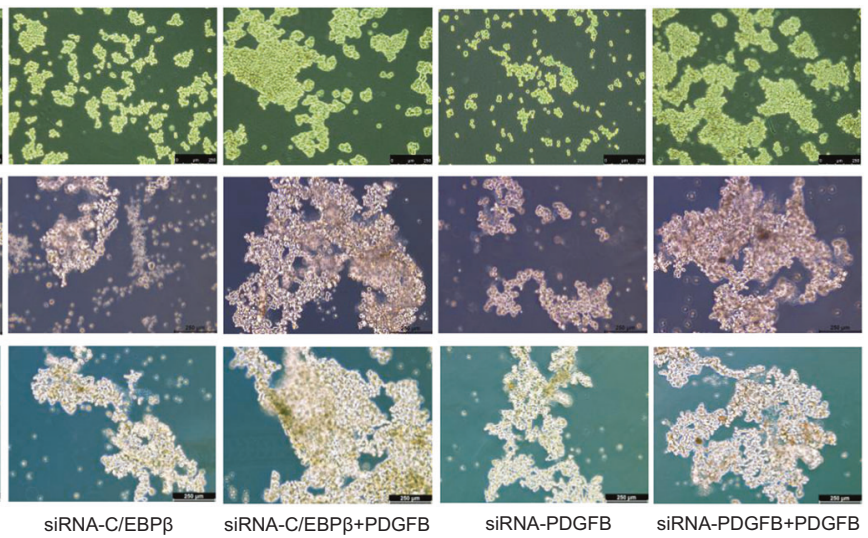

The original article has been corrected. 